\title{
Micro-Motion Feature Extraction of a Rotating Target Based on Interrupted Transmitting and Receiving Pulse Signal in an Anechoic Chamber
}

\author{
Feng Zhao, Xiaobin Liu* ${ }^{\mathbb{C}}$, Zhiming Xu, Yuan Liu and Xiaofeng Ai \\ State Key Laboratory of Complex Electromagnetic Environmental Effects on Electronics and Information System, \\ National University of Defense Technology, Changsha 410073, China; zhaofeng321@nudt.edu.cn (F.Z.); \\ zmxu_nudt@163.com (Z.X.); liuyuan17a@nudt.edu.cn (Y.L.); anxifu2001@163.com (X.A.) \\ * Correspondence: liuxiaobin12@nudt.edu.cn; Tel.: +86-1550-748-6086
}

Received: 4 September 2019; Accepted: 10 September 2019; Published: 13 September 2019

\begin{abstract}
The pulse signal is widely used in micro-motion feature extraction of rapidly rotating targets as its pulse repetition frequency (PRF) can be high. However, when the pulse signal is implemented in a range-limited anechoic chamber for micro-motion feature extraction, the transmitted and reflected pulse signals may be coupled at the receiver. To solve this problem, the interrupted transmitting and receiving (ITR) method is applied to transmit the pulse signal with hundreds of sub-pulses. The target echo can be received when the sub-pulse is not transmitted. Hence, it avoids the coupling effect of transmitted signals and echoes. Then, the whole process of micro-motion feature extraction for rotating target is proposed based on the ITR method. At last, the simulations and experiments verify that the rotating target micro-Doppler can be extracted by the ITR pulse signal.
\end{abstract}

Keywords: pulse radar; rotating target; micro-motion feature extraction; interrupted transmitting and receiving (ITR)

\section{Introduction}

Radar target feature extraction is widely investigated for automatic target recognition [1,2]. The micro-motion feature is one of the most important features [3-5]. In the past decades, the micromotion feature measurement in an anechoic chamber has been conducted via the swept frequency signal [6]. Firstly, the target is placed on the turntable at a certain angle. Then, the swept frequency signal is transmitted to the target, and the scattering field data at the specific aspect angle is obtained. The static measurement is conducted with the turntable turning in different aspect angles. Therefore, in order to obtain precise measurement results, the angle interval of the turntable should be small enough which results in a long measurement time. Besides, the pulse repetition frequency (PRF) of the swept frequency signal in [7] is small for micro-motion feature extraction. As a result, the micro-Doppler of the rapidly micro-motional target may be aliasing. Thus, the micro-motion feature cannot be extracted in this case.

Pulse signal is extensively used in target detection and recognition [8]. As a high PRF of the pulse signal can be achieved $[9,10]$, it can be used in the rapid micro-motion feature extraction of micro-motional target. Then, the turntable turns continuously so that the dynamic measurement of micro-motional target can be implemented in the anechoic chamber. For pulse signal, the target has to be located in the unambiguous range so that it can be detected. However, the size of the anechoic chamber is relatively small in comparison with field experiments, and usually smaller than the minimum unambiguous range. Consequently, the pulse signal is difficult to be used in the anechoic chamber measurement directly. The inner reason is that the target echoes are received while the pulse 
is not completely transmitted. At this time, the transmitted and received pulse signal are coupled at the receiver [11], therefore, the received pulse signal is affected by the transmitted signal. Various wave cancellation methods have been discussed, such as acoustic cancellation [12,13] and radar echo cancellation [14]. However, as the transmitted and received pulse signals are relevant, they are difficult to be separated by the echo cancellation.

In order to conduct the micro-motion feature extraction in anechoic chamber with pulse radar signal, the whole procedure of target measurement is proposed in this paper based on the interrupted transmitting and receiving (ITR) $[11,15,16]$. Firstly, the pulse radar signal is transmitted and received in sub-microseconds to obtain the target echoes. Then, the target echo property is discussed in detail. The micro-motion extraction procedure based on time-frequency analysis $[17,18]$ is provided and analyzed with experimental results.

The remainder of the paper is organized as follows. Section 2 discusses the principle of ITR for pulse radar signal and the characteristics of ITR echo. In Section 3, the procedure of micro-motion feature extraction for rotating target is provided based on the ITR echo. In Section 4, both simulation and experiment results in anechoic chamber are provided with different ITR control parameters to validate of the proposed rotating feature extraction method. Finally, some conclusions are drawn in Section 5 .

\section{Characteristics of Interrupted Transmitting and Receiving Echo}

\subsection{Principle of ITR}

As discussed in [11] and [16], ITR means that the antenna is turned on to transmit the pulse signal in sub-microseconds and then interrupted. After that, the other antenna is turned on to receive the echo. Repeating the above procedures for hundreds of times, the whole pulse is fully transmitted. As a result, the complete pulse signal is divided into hundreds of sub-pulses as shown in Figure 1.



Figure 1. Waveform of $\mathrm{p}(\mathrm{t})$.

The ideal signal to control the transmitting and receiving can be expressed as

$$
p(t)=\operatorname{rect}(t / \tau) * \sum_{n=-\infty}^{+\infty} \delta\left(t-n T_{s}\right)
$$

where $\delta(t)$ is the impulse function, $n$ is the pulse number, $T_{s}$ is the period, ${ }^{*}$ represents the convolution operation, and $\operatorname{rect}(t / \tau)$ is the rectangular envelope as follows

$$
\operatorname{rect}(t / \tau)= \begin{cases}1, & |t / \tau|<0.5 \\ 0, & \text { others }\end{cases}
$$

where $\tau$ is restricted to sub-microsecond to ensure the sub-pulse be fully transmitted before it returns to the receiver in anechoic chamber. In practice, $\tau$ should be designed according to the real size of the anechoic chamber at first.

The spectrum of $p(t)$ is

$$
P(f)=\tau f_{s} \sum_{n=-\infty}^{n=+\infty} \sin \mathrm{c}\left(n f_{s} \tau\right) \delta\left(f-n f_{s}\right)
$$

where $f_{s}=1 / T_{s}$, and $\operatorname{sinc}(x)=\sin (\pi x) /(\pi x)$. 


\subsection{Property of ITR Echo}

Assuming the operated pulse signal is linear frequency modulated (LFM) signal and it can be written as

$$
s(t)=\operatorname{rect}\left(\frac{t}{T_{p}}\right) \exp \left[j 2 \pi\left(f_{c} t+\frac{1}{2} \mu t^{2}\right)\right]
$$

where $f_{c}$ is the carrier frequency, $T_{p}$ is the pulse width, $\mu$ is the chirp rate, and the bandwidth is $B=\mu T_{p}$.

The de-chirp reference signal is

$$
s_{r e f}(t)=\operatorname{rect}\left(\frac{t-2 R_{r e f} / C}{T_{r e f}}\right) \exp \left(j 2 \pi\left(f_{c}\left(t-\frac{2 R_{r e f}}{C}\right)+\frac{1}{2} \mu\left(t-\frac{2 R_{r e f}}{C}\right)^{2}\right)\right)
$$

where $R_{r e f}$ is the reference range, $T_{r e f}$ is the pulse width of reference signal, and $C$ is the electromagnetic wave velocity.

After de-chirping with the reference signal, the difference-frequency output is

$$
s_{f}(t)=s_{r}(t) s_{r e f}^{*}(t)
$$

where $s_{r e f}^{*}(t)$ is the conjugation of $s_{r e f}(t)$, and $s_{r}(t)$ is the echo which can be expressed as

$$
s_{r}(t)=\sum_{k=1}^{K} \alpha_{k} s\left(t-2 R_{k} / C\right)
$$

where $K$ is the number of scattering centers, $\alpha_{k}$ is the scattering coefficient, and $R_{k}$ is the distance between the scattering center $k$ and radar.

Then, the ITR echo is obtained as

$$
\begin{aligned}
s_{r_{2}}(t) & =p_{2}(t) \cdot s_{r}(t) \\
& =\left(\operatorname{rect}\left(\frac{t}{\tau}\right) * \sum_{n \rightarrow-\infty}^{+\infty} \delta\left(t-n T_{s}\right)\right) s_{r}(t)
\end{aligned}
$$

The high-resolution range profile (HRRP) of ITR echo can be obtained after fast Fourier transform (FFT)

$$
S_{f_{3}}(f)=\tau f_{s} T_{p} \sum_{k=1}^{K} \alpha_{k} \exp \left(-j \frac{4 \pi f_{c}}{C} R_{k, \Delta}\right) \sum_{n \rightarrow-\infty}^{+\infty}\left(\operatorname{sinc}\left(n f_{s} \tau\right) \sin c\left(T_{p}\left(f-n f_{s}+2 \frac{\mu}{C} R_{k, \Delta}\right)\right)\right)
$$

where $R_{k, \Delta}=R_{k}-R_{\text {ref }}$.

From (9), we can find that the amplitude is related to $\tau, f_{s}$ and $T_{p}$. The second item sinc(.) in the summation operation illustrates that the de-chirp output is the accumulation of different $\operatorname{sinc}(\cdot)$ functions. Besides, there are different orders of fake peaks in HRRP with the frequency interval $f_{s}$ and the distance between the real and fake peaks is $\Delta R=C f_{s} /(2 \mu)$.

Therefore, the real peaks in HRRP can be fully obtained if $L<\Delta R$, where $L$ is the target length. And then

$$
T_{s}<\frac{C}{2 \mu L}
$$

\section{Micro-Motion Feature Extraction of the Rotating Target}

\subsection{The Micro-Doppler of the Rotating Target}

With the increasing complexity of target structure, many targets contain rotating components, such as aircraft propellers. The distance between the scattering centers and radar varies periodically 
with time, which produces the particular Doppler shift. Figure 2 shows the typical rotating target structure. Scattering centers A and B are both located at the line apexes and the location of which remains unchanged with the rotation. According to the scattering center classification [19,20], A and $\mathrm{B}$ are localized scattering centers which usually correspond to the geometric discontinuities such as corners, edge apexes, cone tips, and so on.

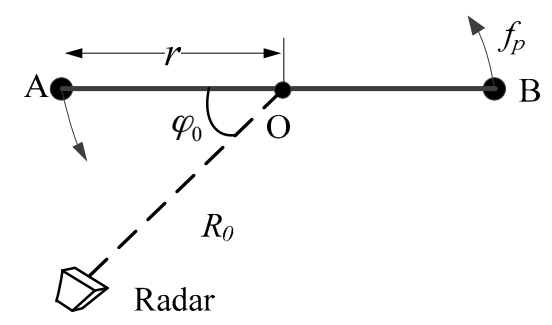

Figure 2. The typical rotating target structure.

Generally, the stop-and-go approximation is adopted, and the position of scattering center is basically unchanged in the fast time. In the slow time $t_{m}$, the distance between point $\mathrm{A}$ and radar can be expressed as

$$
R_{A}\left(t_{m}\right)=R_{0}-r \cos \left(2 \pi f_{p} t_{m}+\varphi_{0}\right)
$$

where $R_{0}$ is the distance between reference scattering center $\mathrm{O}$ and radar, $r$ is the rotation radius, $f_{p}$ is the rotation frequency and $\varphi_{0}$ is the initial phase.

According to (9), the HRRP of the ITR echo including micro-motion information can be obtained as

$$
\begin{aligned}
\hat{S}_{f_{3}}\left(f, t_{m}\right)= & \tau f_{s} T_{p} \sum_{k=1}^{K}\left\{\alpha_{k} \exp \left(-j \frac{4 \pi f_{c}}{\mathrm{C}} R_{k, \Delta}\left(t_{m}\right)\right)\right. \\
& \left.\cdot \sum_{n \rightarrow-\infty}^{+\infty} \operatorname{sinc}\left(n f_{s} \tau\right) \operatorname{sinc}\left[T_{p}\left(f-n f_{s}+2 \frac{\mu}{C} R_{k, \Delta}\left(t_{m}\right)\right)\right] \exp \left(-j \frac{4 \pi n f_{s}}{\mathrm{C}} R_{r e f}\right)\right\}
\end{aligned}
$$

When $n=0$, the peak value of HRRP in (9) is

$$
\hat{S}_{f_{3}}^{\prime}\left(f, t_{m}\right)=\tau f_{s} T_{p} \sum_{k=1}^{K} \alpha_{k} \exp \left(-j \frac{4 \pi f_{c}}{C} R_{k, \Delta}\left(t_{m}\right)\right) \sin c\left[T_{p}\left(f+2 \frac{\mu}{C} R_{k, \Delta}\left(t_{m}\right)\right)\right]
$$

where $R_{k, \Delta}\left(t_{m}\right)=R_{k}\left(t_{m}\right)-R_{r e f}$.

Then, the phase term in (13) is

$$
\phi_{k}\left(t_{m}\right)=-\frac{4 \pi}{\lambda} R_{k, \Delta}\left(t_{m}\right)
$$

where $\lambda=C / f_{c}$ is wavelength.

Therefore, the Doppler frequency of point A is obtained as

$$
f_{\text {micro, } \mathrm{A}}=\frac{1}{2 \pi} \frac{d \phi_{A}\left(t_{m}\right)}{d t_{m}}=\frac{4 \pi r f_{p}}{\lambda} \sin \left(2 \pi f_{p} t_{m}+\varphi_{0}\right)
$$

After time-frequency analysis with (13), the Doppler frequency of point A can be extracted, which is the result of Equation (15).

\subsection{Procedure of the Micro-Doppler Extraction with ITR}

Based on the above analysis, the whole procedure of micro-motion feature extraction for rotating target is given in Figure 3. 


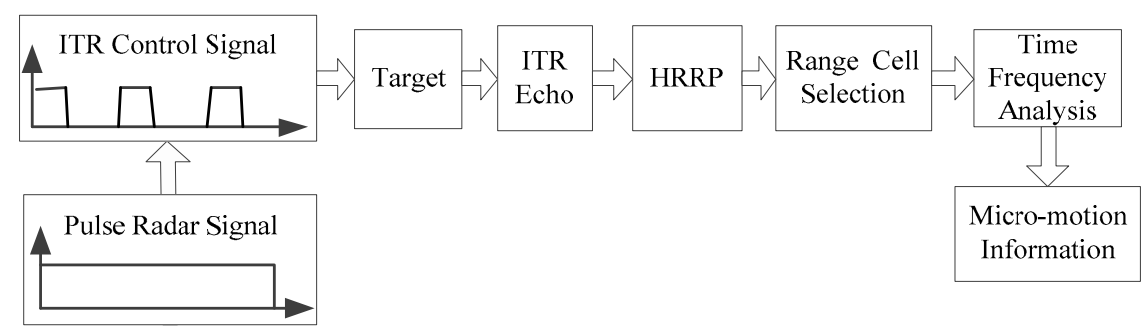

Figure 3. Procedure of micro-motion feature extraction for rotating target.

According to Figure 3, the target echo is received with ITR, and then the target HRRP is obtained by de-chirp processing. After selecting the peak position of target HRRP, the time-frequency analysis can be conducted along the slow time. There are many candidate time-frequency methods such as wavelet transform, Wigner-Ville distribution, short-time Fourier transform (STFT), and so on. Due to simplicity, STFT is chosen in this paper. Finally, the micro-motion information of rotating scattering center can be obtained.

\section{Simulation and Experiment Results}

\subsection{Simulation Results of Rotating Target Micro-Doppler Extraction}

The PRF of typical pulse radar signal may vary from hundreds of Hertz to hundreds of thousands of Hertz $[9,10]$. Therefore, the dynamic measurement of target with high rotation rate can be accomplished by the high PRF of pulse radar signals.

As shown in Figure 2, the typical rotating structure is simplified as two scattering centers, A and B. The distance between the two scattering centers is $2 r=0.2 \mathrm{~m}$. They are rotating around the midpoint $O$ at a frequency of $8 \mathrm{~Hz}$. The slant range between the midpoint $O$ and radar $R_{0}$ is $45 \mathrm{~m}$. The parameters of radar are listed in Table 1.

Table 1. Simulation parameters and values.

\begin{tabular}{cccc}
\hline Parameter & Value & Parameter & Value \\
\hline Pulse width $T_{p}$ & $12 \mu \mathrm{s}$ & Bandwidth $B$ & $500 \mathrm{MHz}$ \\
PRF & $1.67 \mathrm{kHz}$ & ITR period $T_{s}$ & $0.6 \mu \mathrm{s}$ \\
Wave length $\lambda$ & $0.03 \mathrm{~m}$ & ITR pulse width $\tau$ & $0.2 \mu \mathrm{s}$ \\
\hline
\end{tabular}

In Table 1, the bandwidth of the pulse signal and swept frequency signal is $500 \mathrm{MHz}$. As the pulse width is $12 \mu \mathrm{s}$, the distance between the real and fake peaks is $6 \mathrm{~m}$ according to $\Delta R=C f_{s} /(2 \mu)$. Because the target rotation radius is $0.1 \mathrm{~m}$, and the target length is $0.2 \mathrm{~m}$ so that the real and fake peaks can be separated. Moreover, the ITR pulse width $\tau$ should be smaller than $2 R / C$ so that the sub-pulse can be fully transmitted when the reflected signal returns. Because the distance between the target and radar is $R=45 \mathrm{~m}, \tau$ is set as $0.2 \mu$ s which is smaller than $2 R / C=0.3 \mu \mathrm{s}$.

The PRF of swept frequency signal is set as $68 \mathrm{~Hz}$ for comparison since the equivalent PRF of the swept frequency experimental system is $68 \mathrm{~Hz}$ in [7]. The swept frequency interval is $1 \mathrm{MHz}$ and the signal bandwidth is $500 \mathrm{MHz}$.

Firstly, the typical rotating target echo is simulated with swept frequency signal so that the HRRP can be obtained. According to Equations (7) and (8), the complete pulse echoes and ITR echoes are obtained. Then, the corresponding HRRPs are calculated via FFT. At last, the time-frequency analysis is conducted to extract micro-motion features based on the HRRPs. The micro-Doppler extraction results are shown in Figure 4.

Figure $4 \mathrm{a}$ is the range slow-time image obtained by the complete pulse echo. Figure $4 \mathrm{~b}$ is the micro-Doppler extraction result of the complete pulse echo, in which the micro-Doppler frequency is obtained accurately, and it is the ideal result. Figure $4 \mathrm{c}$ is the range slow-time image obtained by 
the swept frequency signal. Figure $4 \mathrm{~d}$ is the micro-Doppler extraction result of the swept frequency signal. Because of the PRF of swept frequency signal is small, i.e., $f_{\text {micro,A }}>\mathrm{PRF} / 2$, the extracted micro-Doppler frequency is aliased. Figure $4 \mathrm{e}$ is the range slow-time image obtained by the ITR echo. According to (10), the ITR period $T_{S}$ is smaller than $18 \mu \mathrm{s}$, therefore, the real peaks can be extracted by adding a suitable interception window in the HRRP. Then, the time frequency analysis is performed to extract the micro-Doppler. We found that the micro-Doppler information depicted in Figure $4 \mathrm{f}$ is basically consistent with that in Figure 4b. In addition, the maximum amplitude values in Figure 4e,f is less than those in Figure $4 \mathrm{a}, \mathrm{b}$ as depicted in the color bar. It is because the echo energy is reduced after ITR. However, the amplitude reduction can be eliminated by energy compensation.

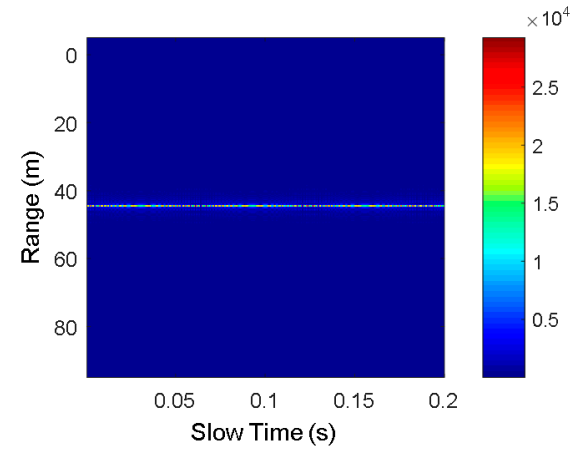

(a)

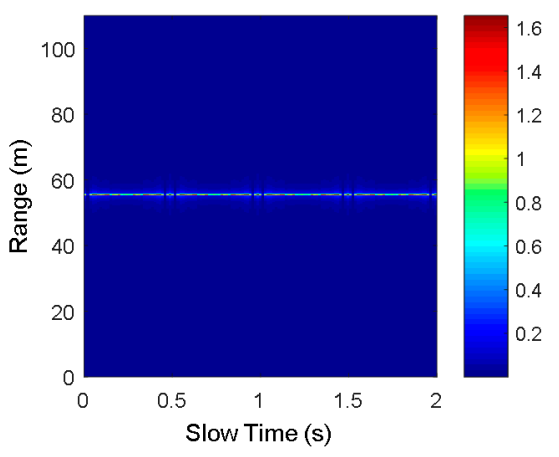

(c)

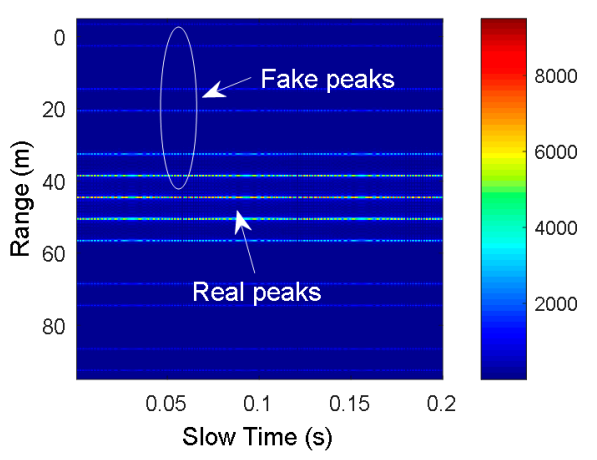

(e)

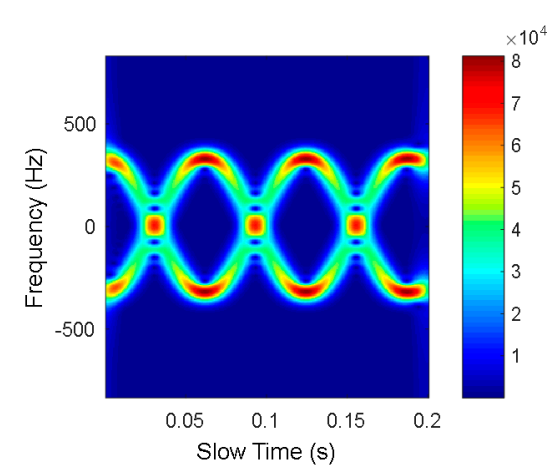

(b)

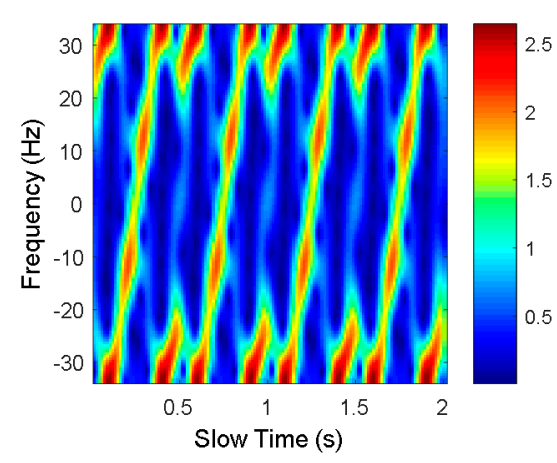

(d)

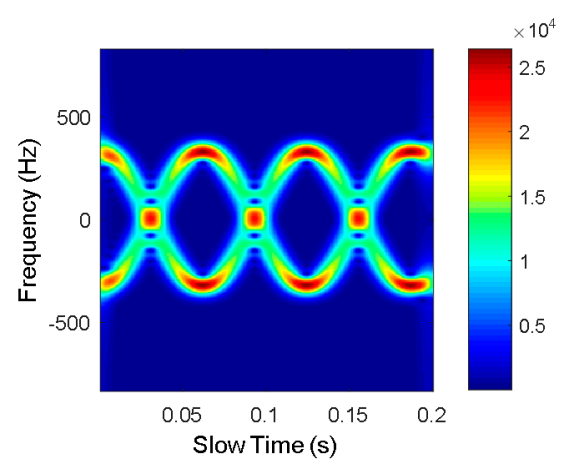

(f)

Figure 4. Micro-Doppler extraction results. (a) Range slow-time image of the complete pulse echo. (b) Time-frequency image of the complete pulse echo. (c) Range slow-time image of the swept frequency echo. (d) Time-frequency image of the swept-frequency echo. (e) Range slow-time image of the ITR echo. (f) Time-frequency image of the interrupted transmitting and receiving (ITR) echo. 


\subsection{Experiment Results in Anechoic Chamber}

In this section, the pulse radar signal ITR experimental system is established for rotating target micro-motion extraction. The experimental system and the rotation target are shown in Figure 5.

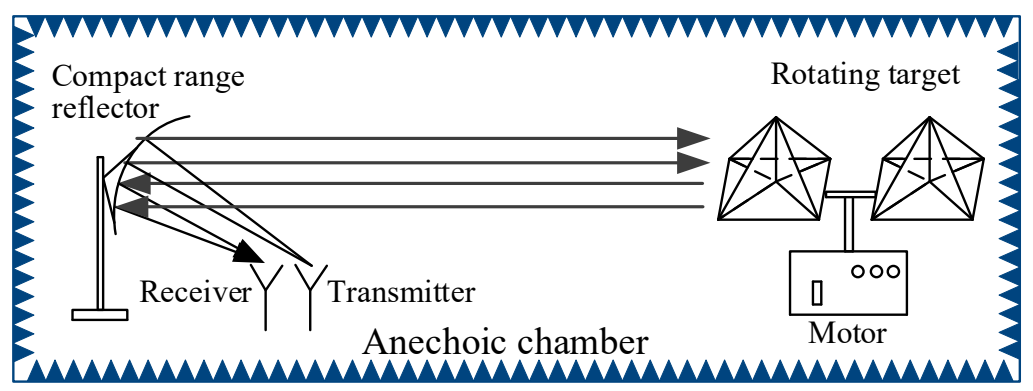

(a) The experiment scenario.



(b) The experimental system. (c) The horn antennas. (d) The rotation target.

Figure 5. Experimental system and rotation target model. (a) The experiment scenario. (b) The experimental system. (c) The horn antennas. (d) The rotation target.

The micro-motion feature measurement experimental scenario is presented in Figure 5a. The compact range reflector is used to ensure the plane wave. The width and length of the anechoic chamber are almost $15 \mathrm{~m}$ and $20 \mathrm{~m}$, respectively. In this case, the pulse width should be smaller than $0.13 \mu \mathrm{s}$ at the least to eliminate the coupling effect of the transmitted and reflected signal. Hence, the use of the ITR method is necessary. As shown in Figure $5 b$, the ITR experimental system is composed of three parts. Part A contains the local oscillator, arbitrary waveform generator (AWG), ITR controller, down-conversion (DC) module and intermediate frequency adjustment module. Part $\mathrm{B}$ is the vector signal generator (VSG) to up-convert the intermediate frequency signal. Part $\mathrm{C}$ is the intermediate frequency digitizer for data acquisition. Figure $5 \mathrm{c}$. shows the transmitter and receiver horn antennas. The intermediate-frequency LFM signal of $300 \mathrm{MHz}$ is generated by the AWG. Then, the signal is up-converted by the VSG to $9.3 \mathrm{GHz}$ and the antenna radiates the signal to target by ITR antenna controller. Target echo is down-converted by the DC module. After intermediate frequency adjustment, the echo can be stored by the intermediate frequency digitizer module. Finally, the ITR echo is processed on the computer.

In Figure 5d, two corner reflectors are placed at the end of the cross bar to simulate the rotating scattering centers. The rotation is controlled by the motor and the rotation rate of the motor needs to be manual controlled during the experiment. The experimental parameters are listed in Table 2.

According to the procedure in Figure 3 , when $B=10 \mathrm{MHz}$ and the rotation rate is about $0.85 \mathrm{~s}$, the results of micro-Doppler extraction are drawn as follows. The maximum rotation frequency of the corner reflector is extracted by time frequency distribution-Hough (TFD-Hough) transformation [21,22].

The HRRP of ITR echo is shown in Figure $6 b$. Then, the range slow-time image can be obtained in Figure 6c. After extracting the target peaks in HRRP, time-frequency analysis can be conducted in the slow time domain to obtain the time-frequency image in Figure $6 \mathrm{~d}$. It can be found that two sinusoidal curves with the same period and opposite initial phases are presented in the time-frequency image, 
which illustrates the micro-Doppler of the two corner reflectors. According to the time-frequency image, the micro-Doppler period is about $0.86 \mathrm{~s}$, which is well consistent with the rotation period $0.85 \mathrm{~s}$ set in the experiment. At the same time, when $\mathrm{r}=0.38 \mathrm{~m}, \lambda=0.03 \mathrm{~m}$, and $f_{p}=1 / 0.85 \mathrm{~s}=1.18 \mathrm{~Hz}$, the theoretical maximum micro-Doppler frequency of the corner reflector is $187.26 \mathrm{~Hz}$ according to (15). The maximum frequency in the time-frequency image is $185.09 \mathrm{~Hz}$, which is also consistent with the calculation results.

Table 2. Parameter setting of uniform transmitting and receiving period for rotating target.

\begin{tabular}{cccc}
\hline Parameter & Value & Parameter & Value \\
\hline Pulse width $T_{p}$ & $12 \mu \mathrm{s}$ & bandwidth $B$ & $10 \mathrm{MHz}$ \\
PRF & $1 \mathrm{kHz}$ & ITR period $T_{s}$ & $300 \mathrm{MHz}$ \\
Wave length & $0.03 \mathrm{~m}(10 \mathrm{GHz})$ & ITR pulse width $\tau$ & $0.1 \mu \mathrm{s}$ \\
Target range & $15.5 \mathrm{~m}$ & rotation radius & $0.38 \mathrm{~m}$ \\
\hline
\end{tabular}

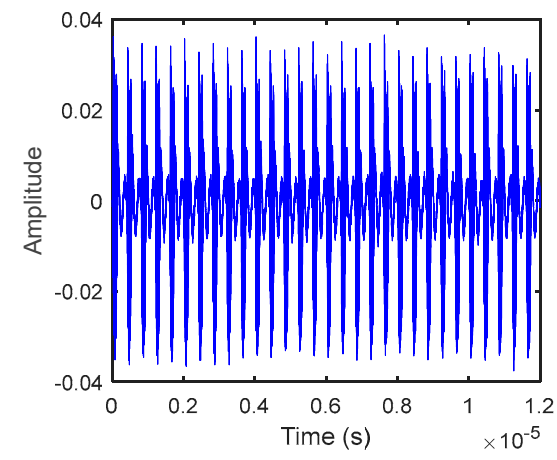

(a)

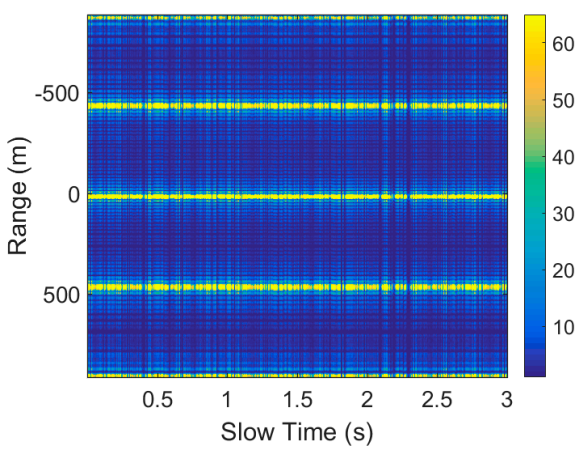

(c)

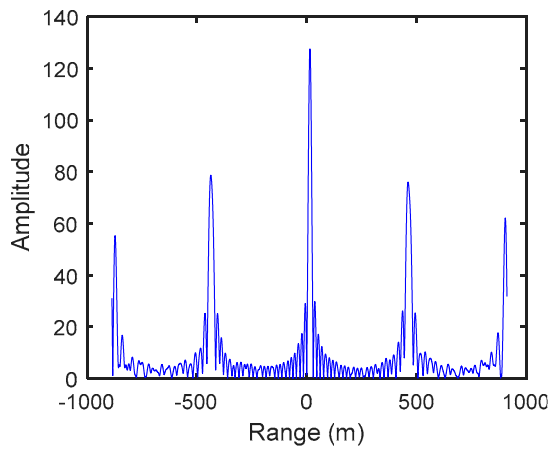

(b)

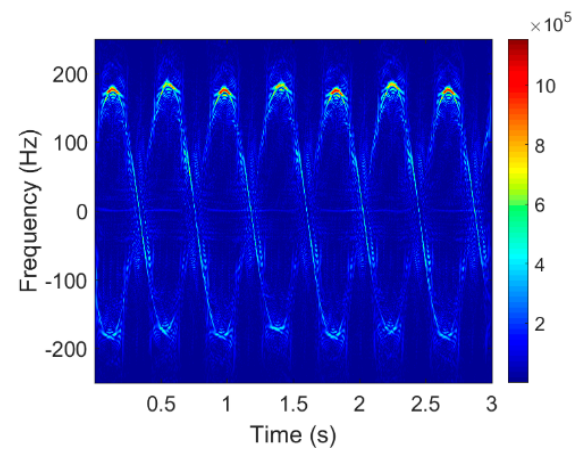

(d)

Figure 6. ITR echo and the micro-Doppler extraction results $(B=10 \mathrm{MHz})$. (a) ITR echo. (b) HRRP of the ITR echo. (c) Range slow-time image. (d) Time-frequency image.

When the rotation period is $1.1 \mathrm{~s}$ and $\mathrm{B}=300 \mathrm{MHz}$, the micro-Doppler extraction results are shown in Figure 7.

The HRRP of ITR echo is shown in Figure $7 \mathrm{~b}$. As the signal bandwidth increases, the range resolution is $C /(2 B)=0.5 \mathrm{~m}$ so that the two reflectors can be distinguished by HRRP in Figure 7c. The range slow-time 2D image is obtained in Figure $7 \mathrm{~d}$,e. It can be seen that the periodic characteristic of HRRP is clear in Figure 7e. When the two reflectors are in the same range cell, the scattering points cannot be separated in the HRRP. Time-frequency analysis is conducted by selecting this range cell and the time-frequency image is obtained as presented in Figure 7f. Comparing Figure 7e,f, when two scattering points are in the same range cell, the corresponding micro-Doppler frequency is maximum. 
In addition, according to the time-frequency image and range slow-time $2 \mathrm{D}$ image, the target rotation period is about $1.122 \mathrm{~s}$ and the maximum micro-Doppler frequency is $141.87 \mathrm{~Hz}$. The rotation rate is set as 1.1s, and the maximum micro-Doppler frequency calculated by (15) is $144.7 \mathrm{~Hz}$, which are all consistent with the experiment results.

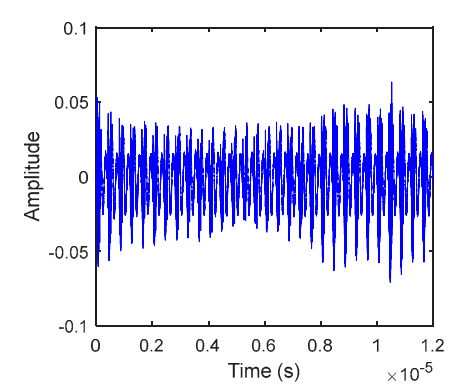

(a)

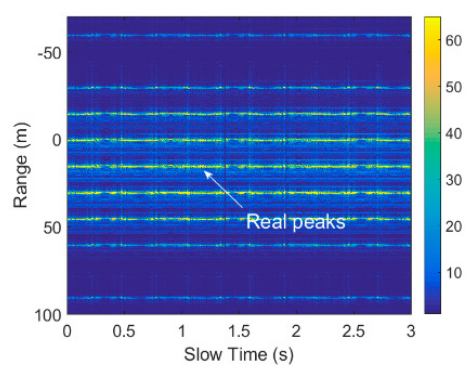

(d)

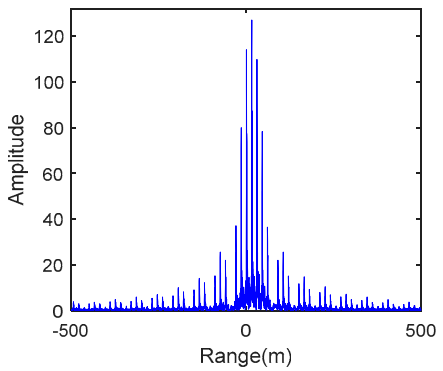

(b)

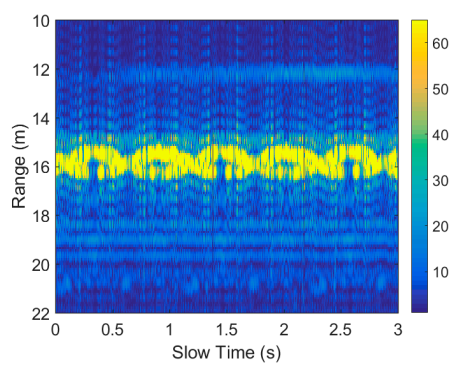

(e)



(c)

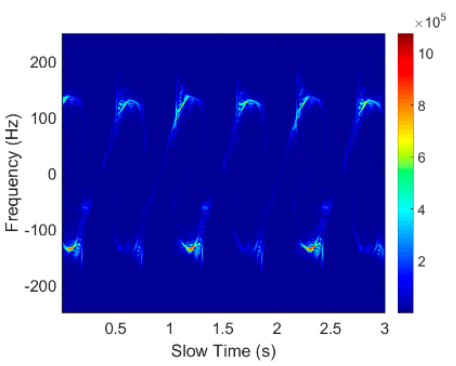

(f)

Figure 7. The ITR echo and micro Doppler extraction results ( $\left.B=300 \mathrm{MHz}, T_{s}=0.4 \mu \mathrm{s}\right)$. (a) The ITR echo. (b) HRRP of ITR echo. (c) Magnification of HRRP. (d) Range slow-time 2D image. (e) Magnification of Range slow-time 2D image. (f) Time-frequency image.

\section{Conclusions}

The whole procedure for the micro-motion feature extraction of rapid micro-motional target is proposed based on the ITR method. The ITR is firstly applied to obtain the target echo in the anechoic chamber. Then, the HRRP of rotating target is deduced with the ITR echo. After that, the target range cell is selected for target micro-Doppler extraction. At last, numerical simulations and experiments in the anechoic chamber are conducted, which verify the validity of the proposed method.

In future, the dynamic measurements of more complicated micro-motional target will be discussed with the proposed method. We will also investigate the echo reconstruction method which makes the ITR echoes approximate the complete pulse echoes as much as possible.

Author Contributions: Methodology, F.Z. and X.L.; software, Z.X.; validation, X.A. and X.L.; formal analysis, F.Z.; investigation, F.Z.; resources, X.A.; data curation, X.L.; writing-original draft preparation, F.Z. and X.L.; writing-review and editing, Z.X. and Y.L.; funding acquisition, F.Z.

Funding: This research was funded by the National Natural Science Foundation of China, grant number 61890542.

Conflicts of Interest: The authors declare no conflict of interest.

\section{References}

1. Wang, Y.; Wang, Q.; Chen, D. Research on target recognition based on edge features. In Proceedings of the 2012 5th International Congress on Image and Signal Processing, Chongqing, China, 16-18 October 2012; pp. 1312-1315. 
2. Gaikwad, A.N.; Singh, D.; Nigam, M.J. Recognition of target in through wall imaging using shape feature extraction. In Proceedings of the 2011 IEEE International Geoscience and Remote Sensing Symposium, Vancouver, BC, Canada, 24-29 July 2011; pp. 957-960.

3. Chen, V.C.; Li, F.; Ho, S.; Wechsler, H. Micro-Doppler effect in radar: Phenomenon, model, and simulation study. IEEE Trans. Aerosp. Electron. Syst. 2006, 42, 2-21. [CrossRef]

4. Zhi, X.; Ai, X.; Wu, Q.; Zhao, F.; Xiao, S. Micro-Doppler characteristics of streamlined ballistic target. Electron. Lett. 2019, 55, 149-157.

5. Ai, X.; Xu, Z.; Wu, Q.; Liu, X.; Xiao, S. Parametric Representation and Application of Micro-Doppler Characteristics for Cone-shaped Space Targets. IEEE Sens. J. 2019. [CrossRef]

6. Liu, J.; Wu, Q.; Ai, X. Experimental study on full-polarization micro-Doppler of space precession target in microwave anechoic chamber. In Proceedings of the IEEE Sensor Signal Processing for Defence, Edinburgh, UK, 22-23 September 2016; pp. 1-5.

7. Liu, J.; Li, G.; Ma, L.; Hu, W.; Li, Y.; Wang, X. Dynamic measurement of micro-motion targets in microwave anechoic chamber. In Proceedings of the IEEE International Radar Conference, Guilin, China, 20-22 April 2009; pp. 1-4.

8. Lin, C.; Tian, R.; Bao, Q.; Chen, Z. A wavelet based denoising method for weak target detection of pulse compression radar. In Proceedings of the Progress in Electromagnetics Research Symposium-Spring (PIERS), St. Petersburg, Russia, 22-25 May 2017; pp. 244-249.

9. Mark, A. Richards, Fundamentals of Radar Signal Processing; McGraw-Hill: New York, NY, USA, 2005.

10. Skolnik, M. Radar Handbook; McGraw-Hill: New York, NY, USA, 2008.

11. Liu, X.; Liu, J.; Zhao, F.; Ai, X.; Wang, G. An equivalent simulation method for pulse radar measurement in anechoic chamber. IEEE Geosci. Remote Sens. Lett. 2017, 14, 1081-1085. [CrossRef]

12. Arun, A. Targeted sound attenuation capacity of 3D printed noise cancelling waveguides. Appl. Acoust. 2019, 151, 30-44.

13. Arun, A. Acoustic absorption of passive destructive interference cavities. Mater. Today Commun. 2019, 19, 68-75.

14. Huang, Q.; Xu, Y. Active cancellation stealth analysis based on interrupted-sampling and convolution modulation. Optik Int. J. Light Electron. Opt. 2016, 127, 3499-3503. [CrossRef]

15. Liu, X.; Liu, J.; Wu, Q.; Zhao, F.; Wang, G. Experimental study on pulse radar target probing in RFS based on interrupted transmitting and receiving. Chin. J. Aeronaut. 2018, 31, 575-583. [CrossRef]

16. Liu, X.; Liu, J.; Zhao, F.; Ai, X.; Wang, G. A novel strategy for pulse radar HRRP reconstruction based on randomly interrupted transmitting and receiving in radio frequency simulation. IEEE Trans. Antennas Propag. 2018, 66, 2569-2580. [CrossRef]

17. Liu, L.; Wang, S.; Zhao, Z. Radar waveform recognition based on time-frequency analysis and artificial bee colony-support vector machine. Electronics 2018, 7, 59. [CrossRef]

18. Guo, K.; Qu, Q.; Feng, A.; Sheng, X. Miss distance estimation based on scattering center model using time-frequency analysis. IEEE Antennas Wirel. Propag. Lett. 2016, 15, 1012-1015. [CrossRef]

19. Guo, K.; Qu, Q.; Sheng, X. Geometry Reconstruction Based on Attributes of Scattering Centers by Using Time-Frequency Representations. IEEE Trans. Antennas Propag. 2016, 64, 708-720. [CrossRef]

20. Xu, Z.; Ai, X.; Wu, Q.; Zhao, F.; Xiao, S. Coupling Scattering Characteristic Analysis of Dihedral Corner Reflectors in SAR Images. IEEE Access 2018, 6, 78918-78930. [CrossRef]

21. Barbaross, S.; Lemoine, O. Analysis of nonlinear FM signals by pattern recognition of their time-frequency representation. IEEE Signal Process. Lett. 1996, 3, 112-115. [CrossRef]

22. Ai, X.; Zou, X.; Yang, J.; Liu, J.; Li, Y. Feature extraction of rotation target based on bistatic micro-Doppler analysis. In Proceedings of the 2011 IEEE CIE International Conference on Radar, Chengdu, China, 24-27 October 2011; pp. 609-612.

(C) 2019 by the authors. Licensee MDPI, Basel, Switzerland. This article is an open access article distributed under the terms and conditions of the Creative Commons Attribution (CC BY) license (http://creativecommons.org/licenses/by/4.0/). 